\title{
TEMAS EMERGENTES EM GESTÃO DE PESSOAS: UMA ANÁLISE DA PRODUÇÃO ACADÊMICA
}

\author{
EMERGING ISSUES IN HUMAN RESOURCES MANAGEMENT: \\ AN ANALYSIS OF ACADEMIC PRODUCTION
}

Recebido - 21/12/2010

Aceito - 14/07/2011

\begin{abstract}
Leilianne Michelle Trindade da Silva Barreto ${ }^{1}$, Maira Patricia da Silva ${ }^{2}$, André Luiz Fischer ${ }^{3}$, Lindolfo Galvão de Albuquerque ${ }^{4}$ e Wilson Aparecido Costa de Amorim ${ }^{5}$
\end{abstract}

\begin{abstract}
Resumo
Este estudo resulta de uma investigação da produção acadêmica nacional e internacional sobre oito temas emergentes em gestão de pessoas: gestão estratégica de pessoas, gestão da diversidade, gestão de talentos, gestão de pessoas internacional, aprendizagem organizacional, responsabilidade social, gestão de gerações e modalidades de trabalho flexível. Esses temas destacaram-se em uma revisão prévia da literatura, que analisou os estudos e as pesquisas sobre tendências, na área, publicados nos últimos cinco anos em importantes fontes, combinada à discussão com especialistas. A análise dos artigos investigados mostrou os temas gestão de pessoas internacional, gestão estratégica de pessoas, aprendizagem organizacional e gestão da diversidade, respectivamente, como os mais frequentes dentro de método utilizado. Quanto aos aspectos metodológicos, a abrangência analítica mais frequente foi a nacional; a natureza da maioria das pesquisas investigadas era exploratória; o perfil metodológico predominante, nos estudos, foi o teóricoempírico; e a abordagem predominante, a quantitativa.
\end{abstract}

Palavras-chave: Gestão de pessoas. Gestão de recursos humanos. Produção acadêmica. Tendências.

1 Doutoranda em Administração FEA - USP. E-mail: leilianne@usp.br

Graduanda em Administração FEA - USP. E-mail: mairapatrici@gmail.com

Professor Doutor de Administração FEA - USP. E-mail: afischer@usp.br

4 Professor Doutor de Administração FEA - USP. E-mail: Igdalbuq@usp.br

5 Doutor em Administração e Coordenador de Pesquisas PROGEP - FIA. E-mail: wilsonamorim@bighost.com.br 


\begin{abstract}
This study shows outcomes from a research about national and international academic production about eight emergent human resource themes: strategic human resource management, diversity management, talent management, international human resource management, organizational learning, social responsibility, generation management, and kinds of flexible job. This group of themes were defined by a previous bibliography revision. This revision included studies and researches published at the last five years about human resource trends at important journals and events. The outcomes were combined with specialist opinions. The analysis identified that international human resource management, strategic human resource management, organizational learning, diversity management were the most common themes. The study also identified that under the methological point of view, the bigger part of the researches were exploratory; the most common methological studies profile were theoretical and empirical and the quantitative approach was predominant.
\end{abstract}

Keywords: People management. Human resource management. Academic production. Trends.

\title{
1 INTRODUÇÃO
}

Para Deadrick e Gibson (2009) a análise da natureza das publicações em periódicos relacionados é uma forma de avaliar o desenvolvimento de um campo. As autoras realizaram essa análise, na área da gestão de pessoas, investigando mais de 6.300 artigos, publicados em quatro periódicos voltados para o campo ao longo de um período de 30 anos (1976-2005). Essa análise longitudinal identificou tendências globais em áreas de interesse da gestão de pessoas, e as questões que dominaram o campo durante o passado e o presente.

As tendências globais da área de gestão de pessoas foram divididas em dois grandes grupos: os temas tradicionais, associados às atividades funcionais de gestão de pessoas; e os temas emergentes, associados aos assuntos contemporâneos desta gestão, que surgem orientados para os eventos atuais. Como temas tradicionais, é possível listar aspectos como motivação, compensação, desempenho humano, desenvolvimento, aspectos legais e relações de trabalho. Como temas emergentes, estão a gestão estratégica de pessoas, a diversidade, a internacionalização da gestão de pessoas, o foco em resultados organizacionais, a tecnologia e o trabalho em equipe. As autoras observaram que, ao longo do tempo, houve um declínio no interesse em temas tradicionais e um aumento no interesse em temas emergentes.

Esses temas são relevantes, e alguns deles estão contemplados nos trabalhos de diversos autores que investigam tendências em gestão de pessoas, os quais também permitem explorar temas complementares. Morley (2004), por exemplo, destaca a aprendizagem organizacional como um fator estratégico para a gestão de pessoas e para a eficiência organizacional. Para o autor, o desenvolvimento dos recursos humanos é preponderante para a as organizações inseridas na atual sociedade do conhecimento. Morley também salienta a necessidade de promover modalidades de trabalho flexíveis, para permitir um melhor atendimento das demandas da vida social dos trabalhadores.

A gestão de talentos também está se tornando um objetivo estratégico para as organizações, devido à elevada demanda por trabalhadores qualificados, à mobilidade da força de trabalho e ao surgimento de ferramentas de recrutamento eletrônico (SHRM, 2007). A preocupação com a responsabilidade social e ambiental também tem sido evidenciada como uma tendência entre as organizações contemporâneas (EGRI; RALSTON, 2008), assim como a gestão da integração entre as diferentes gerações que convivem no ambiente de trabalho (EUROFOUND, 2009-2012). 
A prévia revisão da literatura permitiu enfatizar oito temas emergentes em gestão de pessoas, por aparecerem de forma recorrente nos estudos e pesquisas sobre tendências na área, a saber: gestão estratégica de pessoas, gestão da diversidade, gestão de talentos, gestão de pessoas internacional, aprendizagem organizacional, responsabilidade social, gestão de gerações e modalidades de trabalho flexível.

Dessa forma, este estudo se propõe a investigar a produção acadêmica nacional e internacional sobre esses oito temas emergentes, a partir da análise dos artigos publicados nos últimos cinco anos em importantes fontes da área de gestão de pessoas. Para tanto, introduz-se o referencial teórico sobre os referidos temas, apresenta-se o percurso metodológico seguido para a realização da pesquisa e analisam-se os artigos coletados com base em categorias prédefinidas, encerrando-se com algumas considerações finais.

\section{REFERENCIAL TEÓRICO}

No campo da gestão estratégica de pessoas, merece destaque o artigo de Lengnick-Hall et al. (2009), uma ampla revisão bibliográfica que selecionou artigos que tiveram influência significativa no desenvolvimento do campo nos últimos 30 anos e identificou, assim, um conjunto de sete temas que refletem as tendências e direções que os pesquisadores têm tomado. Os temas investigados são: 1) alinhamento e perspectivas contingenciais; 2) mudança de um foco sobre a gestão de pessoas para a criação de contribuições estratégicas; 3) elaboração de estrutura e componentes do sistema de $\mathrm{RH}$; 4) expansão do escopo da gestão estratégica de pessoas; 5) consecução da execução e implementação de RH; 6) mensuração dos resultados da gestão de pessoas; e 7) avaliação de questões metodológicas.

Os autores afirmam que, durante o estágio embrionário da gestão estratégica de pessoas, três áreas de investigação dominaram a pesquisa no campo. Os estudos iniciais enfatizaram o alinhamento e as perspectivas contingenciais, com o intuito de relacionar as políticas e práticas de gestão de pessoas a vários elementos estratégicos. Ao longo do tempo, essa área de investigação se expandiu, incluindo modelos competitivos para avaliar o alinhamento e considerando os diferentes contextos como fatores de contingência. Em uma segunda linha, os estudos iniciais também trabalharam bastante com a ênfase do RH como uma fonte de contribuições estratégicas importantes, saindo de um foco exclusivo sobre a gestão de pessoas tradicional. Ao longo do tempo, esse campo explorou as contribuições estratégicas das práticas de gestão de pessoas com uma forte fundamentação na visão da empresa baseada em recursos (resource-based view of the firm), incluindo também as contribuições do capital humano e social. Esses temas continuaram a moldar a agenda de pesquisa em gestão estratégica de pessoas no início dos anos 1980. A terceira área de investigação presente neste período de desenvolvimento se refere à expansão do escopo das atividades da gestão estratégica de pessoas para além das convencionais fronteiras organizacionais. Ao longo do tempo, a ênfase nessa área se dirigiu para as preocupações referentes à gestão estratégica de pessoas internacional.

No início dos anos 1990, emergiram dois outros temas. Um se refere à elaboração de estrutura e componentes da gestão de pessoas, abordando a construção da arquitetura de RH e o desenvolvimento de sistemas de trabalho de alto desempenho. O outro enfatiza a importância da efetiva execução das políticas e práticas de $\mathrm{RH}$ e a garantia de realização do intento estratégico. No final dos anos 1990, com o estabelecimento do campo, emergiu uma maior preocupação com a mensuração dos resultados das atividades de gestão estratégica de pessoas. Nos anos 2000, há uma maior maturidade da área, e a metodologia tem ganhado atenção. 
O artigo de Lepak e Shaw (2008) também proporciona uma visão abrangente do tema. Os autores discutem o significado da gestão estratégica de pessoas, diferenciando as pesquisas nesta área das pesquisas sobre a gestão de pessoas tradicional a partir de três aspectos: 1) o nível de análise, que tende a ser voltado para as organizações, os estabelecimentos e/ou as unidades de negócio; 2) a ênfase sobre o alinhamento entre a gestão de pessoas e outros fatores organizacionais e/ou sobre o alinhamento entre as próprias práticas de gestão de pessoas; e 3) a ênfase sobre os resultados organizacionais, incluindo desempenho financeiro e percepções gerenciais sobre o desempenho organizacional. Os autores também discutem as três perspectivas teóricas dominantes sob as quais o pensamento sobre a gestão estratégica de pessoas tem se estruturado: a universalista, que defende a existência de melhores práticas de gestão de pessoas, capazes de proporcionar melhores resultados sob qualquer situação; a contingencial, que ressalta a importância do alinhamento externo com fatores de contingência, tais como a estratégia organizacional; e a configuracional, que ressalta a importância do alinhamento interno entre os elementos que compõem o sistema de gestão de pessoas. Lepak e Shaw destacam a adoção da perspectiva configuracional, que deve levar em consideração o fato de que uma determinada prática de gestão de pessoas, independentemente de sua superioridade em dada situação, dificilmente terá sucesso se não for combinada com outras práticas eficazes. Assim, o alinhamento das práticas é de fundamental importância para que os resultados sejam atingidos. Para alcançar este alinhamento, é importante que os resultados de nível individual das práticas de gestão de pessoas sejam considerados pelas organizações, de forma a contribuir para o conhecimento das sinergias que existem internamente entre as práticas.

A gestão da diversidade também vem ganhando cada vez mais atenção, especialmente em função do crescimento de fenômenos como a globalização e a internacionalização. Nesse sentido, Nishii e Özbilgin (2007) defendem que a melhor maneira para gerenciar as diferenças entre os países é adaptar as políticas organizacionais para os diversos contextos nacionais, pois algumas políticas podem ser eficazes em um país, mas não em outros. Esse raciocínio se aplica também às políticas de gestão de pessoas, que devem ser flexíveis, para conseguir lidar com as diferenças. Para os autores, a falta de adaptação de programas de diversidade, quando levados a outros países, é o principal motivo de falha destes programas, o que indica uma grande necessidade de observação das diferenças demográficas e culturais. Outro problema apontado é que somente parte das empresas costuma engajar seus funcionários estrangeiros em programas de fidelidade ou desenvolver um clima organizacional inclusivo.

De modo similar, Shen et al. (2009) argumentam que a gestão da diversidade pode proporcionar maior produtividade e constituir fonte de vantagem competitiva para as organizações. Segundo os autores, a força de trabalho diversa aumenta a eficiência, a eficácia e a lucratividade das organizações, contribuindo para o seu sucesso. Os autores salientam que, para alcançar esses benefícios oriundos da diversidade, é necessário realizar a sua gestão efetiva, através do uso de estratégias apropriadas de gestão de pessoas. Tais estratégias devem estimular a criação de conhecimento, o aumento da aprendizagem organizacional, a flexibilidade e o desenvolvimento de um ambiente de trabalho que promova a diversidade.

A globalização e a internacionalização exercem influência sobre vários outros aspectos da gestão de pessoas, a exemplo da gestão de talentos e da gestão de pessoas internacional. A gestão de talentos torna-se uma tarefa cada vez mais difícil com a redução das fronteiras e o aumento da mobilidade da força de trabalho. Atualmente, as organizações competem globalmente em seus mercados e também por talentos, esforçando-se para conseguir funcionários mais talentosos (LOSEY; MEISINGER; ULRICH, 2005).

Dentre as estratégias potenciais para a retenção de talentos, é possível citar a possibilidade de crescimento dentro da empresa, a oportunidade de crescimento salarial, a satisfação 
com o trabalho, a flexibilidade do trabalho e a oferta de recompensas contingentes (HAUSKNECHT; RODDA; HOWARD, 2009).

As organizações globalizadas demandam ainda uma gestão de pessoas internacional, que atribui um sentido mais amplo para a função de gestão de pessoas, de modo a dar cobertura às novas questões que aparecem devido à globalização e à internacionalização. Assim, a gestão de pessoas deve repensar muitas das suas práticas tradicionais, de modo a ajudar as organizações a gerenciar e controlar a sua força de trabalho global ao longo de uma variedade de contextos sociais e culturais. Nesse contexto, um importante desafio que se coloca é a gestão de pessoas manter simultaneamente a coerência interna de um sistema sinérgico e adaptá-lo às condições de cada contexto local e regional da organização (MARTíN-ALCÁZAR; ROMEROFERNÁNDEZ; SÁNCHEZ-GARDEY, 2008). Friedman (2007) também apresenta algumas demandas que se colocam para a gestão de pessoas das organizações internacionalizadas: auxiliar a gestão em processos de fusões e aquisições internacionais; equilibrar a necessidade de transferir melhores práticas do país de origem com a necessidade de adotar práticas locais; criar um alinhamento ao longo de fronteiras, culturas e linguagens; entre outros.

Outro tema que tem atraído atenção dos pesquisadores é a aprendizagem organizacional, que também abrange aspectos relacionados à gestão do conhecimento e à educação corporativa. O trabalho de London e Sessa (2007), por exemplo, apresenta um modelo que sugere formas de diagnosticar a aprendizagem em grupos. Este modelo inclui três formas de aprendizagem: a aprendizagem adaptativa, que tem um caráter reativo aos estímulos recebidos, com a realização de pequenas alterações no processo e resultado; a aprendizagem generativa, que é próativa e visa o conhecimento profundo; e a aprendizagem transformativa, que procura mudar a forma como o grupo opera, reconstruindo significados.

Com foco na gestão do conhecimento, Scholl et al. (2004) traçam um panorama sobre a evolução do campo em um horizonte de dez anos. Os resultados indicam que a questão teórica mais insistente e desafiadora para o entendimento e avanço da gestão do conhecimento é a integração dessa gestão com os demais processos do negócio, sendo que algumas abordagens teóricas apontadas como mais eficientes para lidar com esse desafio são o trabalho inter e transdisciplinar e os resultados de pesquisas empíricas sobre o assunto.

A responsabilidade corporativa é evidenciada no estudo de Egri e Ralston (2008), que investigaram as publicações acadêmicas sobre esta temática. Ao averiguar o nível de ênfase que vem sendo dada à investigação do tema nos periódicos, constatou-se que 6,9\% dos 4.671 artigos analisados apresentam a responsabilidade corporativa como tema central. Com relação ao conteúdo, a maior parte dos trabalhos versa sobre ética (37\%) e governança corporativa (25\%), seguidos de responsabilidade ambiental (19\%) e responsabilidade social (18\%). Verificou-se também que $75 \%$ desses artigos apresentam abordagem empírica, e somente 25\%, teórica. Dentre os países mais estudados estão Estados Unidos, China, Holanda, Inglaterra, Alemanha, Canadá, Japão e Austrália.

Os estudos realizados nesse campo salientam os benefícios experimentados pelas organizações que desempenham uma postura socialmente responsável e destacam a importância da gestão de pessoas no desenvolvimento de organizações sustentáveis impactando a imagem da organização, de modo a torná-la mais atraente como empregadora, trazendo reflexos sobre o comprometimento dos funcionários (BRAMMER; MILLINGTON; RAYTON, 2007).

A gestão de gerações também tem despertado o interesse dos estudiosos de gestão de pessoas. Com as diversas mudanças demográficas, as organizações passaram a se preocupar com a integração entre os diversos grupos geracionais no local de trabalho, gerindo os conflitos advindos das diferenças na percepção e compreensão dos valores éticos entre as gerações e suas diferentes necessidades e formas de trabalho (EUROFOUND, 2009-2012). 
Nos próximos anos, a gestão de pessoas deve focar mais a preparação de uma nova geração de trabalhadores, enquanto ajuda os trabalhadores mais velhos a permanecer no trabaIho ou na transição para a aposentadoria. O desenvolvimento de competências, através de iniciativas de treinamento, educação e aprendizagem, e a atração e retenção de trabalhadores altamente qualificados, onde quer que eles estejam localizados no mundo, estão entre as formas mais comuns com que os profissionais de gestão de pessoas planejam responder a essas mudanças (SHRM, 2008).

Já as modalidades de trabalho flexíveis têm sido tratadas pelos profissionais da gestão de pessoas como formas de atender à necessidade dos funcionários de alcançar um equilíbrio entre trabalho e vida pessoal e familiar. Tais modalidades envolvem: trabalho de meio-período, teletrabalho, homework, redução da jornada de trabalho semanal, flexibilização dos horários de trabalho, trabalho por turnos, contratos com limite de tempo, entre outros. Assim, a flexibilidade da jornada de trabalho surge como modo de contribuir para a conciliação de obrigações (KERSLEY et al., 2005).

Apesar de a oferta de modalidades de trabalho flexíveis partir do objetivo de equilibrar trabalho e vida pessoal, ela deve ser desenvolvida nas organizações com bastante cautela. Lautsch e Scully (2007) destacam que os dilemas sobre as horas de trabalho exigem a busca por soluções mais minuciosas dentro da organização, além de políticas e mobilizações sociais mais amplas. Para os autores, as políticas de flexibilização que envolvem simples reduções de tempo de trabalho, para que o funcionário trabalhe menos mas receba remuneração proporcional, não funcionam efetivamente, pois causam impactos negativos sobre as finanças da família.

\section{METODOLOGIA}

\subsection{Eixos temáticos}

Com o objetivo de discutir temas emergentes em gestão de pessoas, foi realizada, em estudo anterior, uma ampla revisão bibliográfica, com o propósito de investigar artigos que tratavam de tendências e do estado da arte em gestão de pessoas.

A partir dessa pesquisa prévia, foram apontados 11 temas recorrentes que a literatura indicava como tendências para a área de gestão de pessoas. Estes temas foram submetidos à análise e ao julgamento de especialistas da área, os quais, consensualmente, elegeram 8 eixos temáticos de maior relevância para os próximos anos, que merecem uma investigação mais profunda, a saber: 1) gestão estratégica de pessoas: envolve literatura sobre gestão estratégica de modo geral, comprometimento organizacional, avaliação de resultados, gestão da mudança e consultoria interna; 2) gestão da diversidade: envolve a gestão da diversidade nas organizações, as questões de inclusão no mercado de trabalho, as diferenças de gênero etc.; 3) gestão de talentos: envolve a literatura sobre atração e retenção de talentos; 4) gestão de pessoas internacional: envolve a literatura de gestão estratégica internacional de pessoas, gestão de expatriados, gestão global de pessoas etc.; 5) aprendizagem organizacional: envolve a literatura de educação corporativa, aprendizagem organizacional e gestão e transferência do conhecimento; 6) responsabilidade corporativa: envolve a literatura de responsabilidade social e ambiental e sustentabilidade; 7) gestão de gerações: envolve a gestão de trabalhadores mais velhos, gestão de segundas carreiras e gestão da nova geração no mercado de trabalho; e 8) modalidades de trabalho flexíveis: envolve a literatura sobre flexibilização de horas e contratos de trabalho, o teletrabalho etc. 
Destaca-se que essa ordem dada aos temas se repetirá por todo o artigo, servindo como legenda para as tabelas que se apresentam de forma resumida, apenas com a numeração de um a oito referente aos eixos temáticos estabelecidos.

\subsection{Horizonte de tempo}

O levantamento da literatura sobre temas emergentes em gestão de pessoas envolveu o horizonte de tempo de cinco anos, tendo sido pesquisados os artigos publicados entre janeiro de 2005 e agosto de 2009. Os trabalhos que realizam pesquisas bibliográficas costumam trabaIhar com um período de dez anos (TONELLI et al., 2003; JABBOUR; SANTOS; BARBIERI, 2008). No entanto, optou-se pela redução do horizonte de tempo, uma vez que o objetivo do trabalho é investigar a literatura sobre tendências recentes em gestão de pessoas.

\subsection{Escolha de periódicos}

A pesquisa bibliográfica contemplou três periódicos internacionais dedicados à área de gestão de pessoas. O critério de seleção envolveu a catalogação pelo Journal Citation Reports (JCR) do Institute for Scientific Information (ISI). Este sistema avalia periódicos e determina o seu fator de impacto, por meio do número de citações de artigos publicados nestes periódicos. Para a área de Management, existem 89 periódicos analisados no JCR de 2008, dos quais dois são voltados para a área de gestão de pessoas: a) o International Journal of Human Resource Management (IJHRM), com fator de impacto de 0,856; e b) o Human Resource Management (HRM), com fator de impacto de 0,729. Genericamente, periódicos com fator de impacto acima de 0,5 (meio) já se incluem na lista Qualis da CAPES como de nível B1 ou A2.

Por meio desse critério, afirma-se que estes representam os dois melhores periódicos de gestão de pessoas a nível mundial. Além deles, decidiu-se incluir outro periódico de destaque na área de gestão de pessoas, que não está contemplado pela avaliação do JCR, mas que é reconhecido pela comunidade acadêmica: o Human Resource Management Review (HRMR) (DEADRICK; GIBSON, 2009). A sua inclusão justifica-se pelo fato de ele ter lançado recentemente (junho de 2009) um número especialmente dedicado a tendências emergentes em gestão de pessoas.

A nível nacional, realizou-se uma pesquisa nos principais periódicos da área de Administração listados pelo Qualis da CAPES. Entretanto, não existe nenhum dedicado especialmente à área de gestão de pessoas, o que implicou uma quantidade ínfima de artigos publicados nos eixos temáticos elencados. Ainda em busca de produção acadêmica de qualidade no Brasil, optou-se pela consulta aos artigos publicados na área de gestão de pessoas dos anais do EnANPAD (Encontro da Associação Nacional de Pós-Graduação e Pesquisa em Administração). Este é o evento brasileiro de maior destaque na área, sendo veículo bastante utilizado por pesquisadores que trabalham com revisão da literatura em Administração (ROSSONI, 2006; HOPPEN; MEIRELLES, 2005; TONELLI et al., 2003).

\subsection{Critérios de seleção de artigos}

Uma vez definidos os periódicos, realizou-se a busca integral em todas as edições no período de cinco anos considerado, selecionando-se os artigos que abordavam os temas prédefinidos. A seleção dos artigos ocorreu primeiramente pelo título e depois pelo resumo. Quando o título indicava que o artigo tratava sobre gestão de pessoas e que trabalhava com um dos 
temas considerados na revisão da literatura, partia-se para a leitura do resumo, para verificar se o objetivo realmente envolvia o tema. Seguindo esses critérios, o quadro 1 apresenta a quantidade de artigos selecionados em cada eixo temático e em cada periódico no período de 2005 a 2009.

\begin{tabular}{|c|c|c|c|c|c|}
\hline $\begin{array}{l}\text { Fonte de Publicação } \\
\text { Eixos temáticos/Ano de publicação }\end{array}$ & IJHRM & HRM & HRMR & ANPAD & TOTAL \\
\hline 1. Gestão estratégica de pessoas & 10 & 7 & 4 & 5 & 26 \\
\hline 2009 & 2 & 1 & 1 & 0 & 4 \\
\hline 2008 & 4 & 5 & 0 & 3 & 12 \\
\hline 2007 & 0 & 0 & 2 & 0 & 2 \\
\hline 2006 & 1 & 1 & 0 & 1 & 3 \\
\hline 2005 & 3 & 0 & 1 & 1 & 5 \\
\hline 2. Gestão da diversidade & 6 & 6 & 1 & 2 & 15 \\
\hline 2009 & 1 & 0 & 1 & 0 & 2 \\
\hline 2008 & 1 & 6 & 0 & 0 & 7 \\
\hline 2007 & 2 & 0 & 0 & 1 & 3 \\
\hline 2006 & 1 & 0 & 0 & 1 & 2 \\
\hline 2005 & 1 & 0 & 0 & 0 & 1 \\
\hline 3. Gestão de talentos & $\mathbf{0}$ & 2 & 1 & 1 & 4 \\
\hline 2009 & 0 & 1 & 0 & 0 & 1 \\
\hline 2008 & 0 & 0 & 0 & 1 & 1 \\
\hline 2007 & 0 & 0 & 0 & 0 & 0 \\
\hline 2006 & 0 & 0 & 1 & 0 & 1 \\
\hline 2005 & 0 & 1 & 0 & 0 & 1 \\
\hline 4. Gestão de pessoas internacional & 22 & 13 & 2 & 2 & 39 \\
\hline 2009 & 7 & 10 & 0 & 0 & 17 \\
\hline 2008 & 3 & 0 & 0 & 1 & 4 \\
\hline 2007 & 5 & 2 & 0 & 1 & 8 \\
\hline 2006 & 2 & 1 & 1 & 0 & 4 \\
\hline 2005 & 5 & 0 & 1 & 0 & 6 \\
\hline 5. Aprendizagem organizacional & 1 & 12 & $\mathbf{0}$ & 5 & 18 \\
\hline 2009 & 0 & 8 & 0 & 0 & 8 \\
\hline 2008 & 0 & 1 & 0 & 4 & 5 \\
\hline 2007 & 1 & 1 & 0 & 0 & 2 \\
\hline 2006 & 0 & 0 & 0 & 1 & 1 \\
\hline 2005 & 0 & 2 & 0 & 0 & 2 \\
\hline 6. Responsabilidade corporativa & 4 & $\mathbf{0}$ & $\mathbf{0}$ & 3 & 7 \\
\hline 2009 & 1 & 0 & 0 & 0 & 1 \\
\hline 2008 & 2 & 0 & 0 & 1 & 3 \\
\hline 2007 & 1 & 0 & 0 & 1 & 2 \\
\hline 2006 & 0 & 0 & 0 & 1 & 1 \\
\hline 2005 & 0 & 0 & 0 & 0 & 0 \\
\hline 7. Gestão de gerações & 1 & o & $\mathbf{0}$ & 2 & 3 \\
\hline 2009 & 0 & 0 & 0 & 0 & 0 \\
\hline 2008 & 0 & 0 & 0 & 1 & 1 \\
\hline 2007 & 1 & 0 & 0 & 0 & 1 \\
\hline 2006 & 0 & 0 & 0 & 0 & 0 \\
\hline 2005 & 0 & 0 & 0 & 1 & 1 \\
\hline 8. Modalidades de trabalho flexíveis & 9 & $\mathbf{0}$ & o & o & 9 \\
\hline 2009 & 3 & 0 & 0 & 0 & 3 \\
\hline
\end{tabular}

Quadro 1 - Artigos selecionados por eixo temático e por periódico entre 2005 e 2009 


\subsection{Categorias de análise}

As categorias de análise foram elencadas com base em estudos que investigam a produção científica na área de Administração (TONELLI et al., 2003; JABBOUR; SANTOS; BARBIERI, 2008; CARDOSO et al., 2005; BRANDÃO, 2008). Assim, os artigos selecionados serão investigados e analisados nas seguintes categorias: 1) temática: classificação segundo os oito eixos temáticos eleitos para a pesquisa; 2) objetivo principal: categorias arbitrárias geradas a partir da listagem dos objetivos; 3) abrangência analítica: multinacional, nacional, regional, setor econômico, organização e área funcional; 4) natureza da pesquisa: exploratória, descritiva e explicativa; 5) perfil metodológico: empírico, teórico-empírico e teórico; 6) abordagem metodológica: qualitativa e quantitativa; 7) estratégia metodológica: survey, estudo de caso, observação e pesquisa bibliográfica; e 8) conteúdo dos trabalhos: listagem das contribuições dos artigos.

\section{ANÁLISE DOS RESULTADOS}

\subsection{Temática}

A pesquisa nos periódicos e eventos acadêmicos buscou artigos sobre os eixos temáticos eleitos. Como ilustra a tabela 1, os temas de maior frequência foram a gestão de pessoas internacional (32\%) e a gestão estratégica de pessoas (21\%), mas as questões sobre aprendizagem organizacional (15\%) e gestão da diversidade (12\%) também tiveram número significativo de artigos. Estes quatro temas podem ser considerados os mais atrativos para os pesquisadores nos últimos cinco anos.

Tabela 1 - Eixos temáticos

\begin{tabular}{|l|r|r|}
\hline \multicolumn{1}{|c|}{ Temas } & Artigos & \multicolumn{1}{c|}{$\%$} \\
\hline 1 - Gestão estratégica de pessoas & 26 & $21 \%$ \\
\hline 2 - Gestão da diversidade & 15 & $12 \%$ \\
\hline 3 - Gestão de talentos & 4 & $3 \%$ \\
\hline 4 - Gestão de pessoas internacional & 39 & $32 \%$ \\
\hline 5 - Aprendizagem organizacional & 18 & $15 \%$ \\
\hline 6 - Responsabilidade corporativa & 7 & $6 \%$ \\
\hline 7 - Gestão de gerações & 3 & $2 \%$ \\
\hline 8 - Modalidades de trabalho flexíveis & 9 & $7 \%$ \\
\hline Total & $\mathbf{1 2 1}$ & $\mathbf{1 0 0 \%}$ \\
\hline
\end{tabular}

Os outros quatro temas apresentaram frequência inferior a 10\% entre os artigos pesquisados. Um número muito pequeno de trabalhos foi encontrado sobre gestão de gerações e gestão de talentos - apenas $2 \%$ e $3 \%$, respectivamente. O mesmo aconteceu com responsabilidade corporativa (6\%) e modalidades de trabalho flexíveis (7\%). A baixa frequência destes temas denota que eles não vêm sendo muito pesquisados nos últimos cinco anos. Isso sugere que eles são temas emergentes e que atrairão a atenção dos estudiosos de gestão de pessoas nos próximos anos ou que eles já foram alvo de atenção em algum momento e estão deixando de ser pesquisados pelo interesse principal em outros temas. 


\subsection{Objetivo principal}

Em função da grande diversidade de temas e assuntos tratados, não foi possível criar categorias genéricas de objetivos suficientemente amplas para abarcar todos os artigos analisados. No entanto, é possível descrever o panorama geral das proposições trazidas pelos artigos explorados em cada uma das temáticas. Os artigos que versavam sobre gestão estratégica de pessoas apresentavam, como objetivo mais frequente, analisar relações entre a gestão de pessoas e os resultados organizacionais, que é a essência da teoria deste campo. Alguns estudos analisaram também variáveis moderadoras dessas relações, tais como a estratégia, a estrutura e a cultura organizacional. Outros investigaram o impacto de práticas específicas da gestão de pessoas, a exemplo do trabalho em equipe. Também foram frequentes artigos que analisaram o desenvolvimento da literatura sobre gestão estratégica de pessoas e que analisaram o comprometimento organizacional e as suas variáveis.

Na temática de gestão da diversidade, os estudos congregam as mais variadas categorias de diversidade, tais como raça, gênero, orientação sexual e outros grupos de minorias. De modo geral, são analisados a integração dos grupos de minorias nos cargos de nível estratégico das organizações, a influência da escassez de habilidades para a abertura de oportunidades para as minorias no mercado de trabalho, o impacto do tamanho da organização sobre o acolhimento dos grupos diversos, entre outros.

Os artigos sobre gestão de talentos abordavam os fatores que contribuem para a retenção de talentos nas organizações, as estratégias de atração e retenção de talentos frente à tendência de escassez de profissionais qualificados no mercado, o comportamento dos funcionários que possuem participação nas ações da empresa e a revisão crítica da literatura sobre a área.

Muitos artigos sobre gestão de pessoas internacional foram encontrados, por isso existe uma grande diversidade de objetivos. Embora de difícil categorização, alguns objetivos comuns podem ser identificados. Dentre eles, os relativos à investigação de fatores ligados ao processo de expatriação e/ou repatriação, como a percepção e as expectativas dos funcionários e os fatores críticos de sucesso. Outros pontos investigados, com significativa frequência, foram os processos de seleção internacionais, as questões ligadas à gestão estratégica internacional, a gestão de equipes internacionais e políticas e as práticas de RH em empresas internacionalizadas.

Os artigos sobre aprendizagem organizacional têm objetivos bastante diversos, mas destacam-se os que dizem respeito à investigação dos fatores que interferem na aprendizagem e os ligados à gestão e transferência do conhecimento nas organizações.

Os estudos sobre responsabilidade corporativa apresentaram três grandes objetivos: analisar o papel da gestão de pessoas no desenvolvimento de organizações sustentáveis, verificar a consistência do discurso das organizações em torno do movimento da responsabilidade social empresarial e investigar o impacto da responsabilidade social sobre as percepções e atitudes dos funcionários.

Os 3 artigos que compõem o quadro da gestão de gerações estão associados às gerações mais velhas, abarcando questões sobre o equilíbrio entre trabalho e vida pessoal de trabalhadores mais velhos, o bem-estar e a qualidade de vida na terceira idade e as necessidades e preferências dos aposentados.

Em relação às modalidades de trabalho flexíveis, os objetivos propostos foram bastante variados, envolvendo aspectos como flexibilidade interna do trabalho, percepções sobre qualidade do trabalho, gestão do conhecimento em organizações com forte presença de profissionais com contratos flexíveis de trabalho, entre outros. 


\subsection{Abrangência analítica}

De forma geral, a abrangência analítica mais frequente, nas pesquisas encontradas, foi a nacional (32\%), mas diversos estudos também abordaram mais de um país (12\%). No entanto, muitos artigos tinham suas análises restritas a uma organização (11\%) ou região (10\%). Não foi possível identificar a abrangência analítica em $23 \%$ dos artigos, especialmente em função da grande presença de estudos teóricos que tratam o tema de forma ampla, sem especificar a abrangência considerada. Conforme a tabela 2, as distribuições de frequência se mantêm constantes também quando separadas por cada um dos eixos temáticos, com destaque para os 29\% de artigos multinacionais de responsabilidade corporativa (6), mais que o dobro do resultado geral. Um resultado surpreendente é o pequeno número de estudos multinacionais (18\%) dentro do tema de gestão de pessoas internacional (4).

Tabela 2 - Abrangência analítica

\begin{tabular}{|l|r|r|r|r|r|r|r|r|r|}
\hline \multirow{2}{*}{ Abrangência analítica } & \multirow{2}{*}{ Geral } & \multicolumn{7}{|c|}{ Eixos temáticos } \\
\cline { 3 - 10 } & & $\mathbf{1}$ & $\mathbf{2}$ & $\mathbf{3}$ & $\mathbf{4}$ & $\mathbf{5}$ & $\mathbf{6}$ & \multicolumn{1}{c|}{7} & $\mathbf{8}$ \\
\hline Multinacional & $12 \%$ & $8 \%$ & $13 \%$ & 0 & $18 \%$ & $6 \%$ & $29 \%$ & 0 & 0 \\
\hline Nacional & $32 \%$ & $42 \%$ & $20 \%$ & $50 \%$ & $31 \%$ & $11 \%$ & $14 \%$ & 0 & $89 \%$ \\
\hline Regional & $10 \%$ & $8 \%$ & $27 \%$ & 0 & $5 \%$ & $6 \%$ & $14 \%$ & $67 \%$ & 0 \\
\hline Setor econômico & $6 \%$ & $8 \%$ & 0 & 0 & $3 \%$ & $11 \%$ & 0 & $33 \%$ & $11 \%$ \\
\hline Organização & $11 \%$ & $12 \%$ & $7 \%$ & $25 \%$ & $5 \%$ & $22 \%$ & $29 \%$ & 0 & 0 \\
\hline Área funcional & $7 \%$ & 0 & 0 & $25 \%$ & $10 \%$ & $17 \%$ & 0 & 0 & 0 \\
\hline Não identificada & $23 \%$ & $23 \%$ & $33 \%$ & 0 & $28 \%$ & $28 \%$ & $14 \%$ & 0 & 0 \\
\hline Total & $\mathbf{1 0 0} \%$ & $\mathbf{1 0 0} \%$ & $\mathbf{1 0 0} \%$ & $\mathbf{1 0 0} \%$ & $\mathbf{1 0 0} \%$ & $\mathbf{1 0 0} \%$ & $\mathbf{1 0 0} \%$ & $\mathbf{1 0 0} \%$ & $\mathbf{1 0 0} \%$ \\
\hline
\end{tabular}

Outra frequência de destaque se refere aos artigos da temática de modalidades de trabalho flexíveis (8), que apresenta $89 \%$ dos artigos conduzidos a nível nacional. Isto pode indicar que este tema ainda é muito peculiar para a realidade de cada país, tornando desnecessária a realização de estudos regionais. Entretanto, a realização de estudos comparativos internacionais, para confrontar as características assumidas nesta área nos diferentes países seria interessante. Entre estudos nacionais e multinacionais realizados nos diversos temas, os principais países estudados foram os Estados Unidos, o Canadá, o continente europeu de forma geral - com destaque para Inglaterra, Espanha, Alemanha e Holanda -, a Índia, a China e o Japão, além dos estudos envolvendo o Brasil.

\subsection{Natureza da pesquisa}

Conforme ilustra a tabela 3, a maioria das pesquisas investigadas apresenta natureza exploratória (59\%), o que pode receber influência da quantidade de trabalhos teóricos e de estudos de casos constantes entre os artigos analisados. Essa natureza predominante também foi utilizada em conjunto com pesquisas descritivas e explicativas. A natureza descritiva se mostrou presente em um número razoável de estudos (27\%) e a pesquisa explicativa teve um percentual muito pequeno (4\%). Além disso, não foi possível identificar a natureza da pesquisa em $4 \%$ dos artigos. 
Tabela 3 - Natureza da pesquisa

\begin{tabular}{|l|r|r|r|r|r|r|r|r|r|}
\hline \multirow{2}{*}{ Natureza da pesquisa } & \multirow{2}{*}{ Geral } & \multicolumn{9}{|c|}{ Eixos temáticos } \\
\cline { 3 - 11 } & & $\mathbf{1}$ & \multicolumn{1}{c|}{$\mathbf{2}$} & \multicolumn{1}{c|}{$\mathbf{3}$} & $\mathbf{4}$ & \multicolumn{1}{c|}{$\mathbf{5}$} & \multicolumn{1}{c|}{} & \multicolumn{1}{c|}{7} & $\mathbf{8}$ \\
\hline Exploratória & $59 \%$ & $38 \%$ & $60 \%$ & $75 \%$ & $87 \%$ & $50 \%$ & $14 \%$ & $67 \%$ & $33 \%$ \\
\hline Descritiva & $27 \%$ & $35 \%$ & $27 \%$ & 0 & $13 \%$ & $28 \%$ & $57 \%$ & $33 \%$ & $56 \%$ \\
\hline Explicativa & $4 \%$ & $12 \%$ & $7 \%$ & $25 \%$ & 0 & 0 & 0 & 0 & $\%$ \\
\hline Exploratório-descritiva & $3 \%$ & $8 \%$ & 0 & 0 & 0 & 0 & $14 \%$ & 0 & $11 \%$ \\
\hline Exploratório-explicativa & $2 \%$ & $4 \%$ & 0 & 0 & 0 & $11 \%$ & 0 & 0 & 0 \\
\hline Não identificada & $4 \%$ & $4 \%$ & $7 \%$ & 0 & 0 & $11 \%$ & $14 \%$ & 0 & 0 \\
\hline Total & $\mathbf{1 0 0} \%$ & $\mathbf{1 0 0} \%$ & $\mathbf{1 0 0} \%$ & $\mathbf{1 0 0} \%$ & $\mathbf{1 0 0} \%$ & $\mathbf{1 0 0} \%$ & $\mathbf{1 0 0} \%$ & $\mathbf{1 0 0} \%$ & $\mathbf{1 0 0} \%$ \\
\hline
\end{tabular}

A maior parte dos eixos temáticos acompanha essa tendência de realização de estudos exploratórios, com exceção dos temas responsabilidade corporativa (6) e modalidades de trabaIho flexíveis (8), que apresentam uma maior parcela de estudos de natureza descritiva - 57\% e $56 \%$, respectivamente. Estes dois temas são também os que apresentam maior frequência de pesquisas exploratório-descritivas. Vale ainda destacar que os temas gestão de talentos (3) e gestão estratégica de pessoas (1) foram os que apresentaram frequências mais elevadas de estudos de natureza explicativa, o que não acontece nos demais eixos temáticos.

\subsection{Perfil metodológico}

O perfil metodológico predominante nos estudos foi o teórico-empírico (71\%), pois a maioria dos artigos mesclava a revisão teórica com a busca de dados e análises empíricas. Nenhum artigo apresentou perfil exclusivamente empírico, o que já era esperado para trabalhos na área de gestão de pessoas que, normalmente, desenvolvem um referencial teórico fundamentado em análises empíricas posteriores. Já o perfil exclusivamente teórico foi utilizado em $35 \%$ dos artigos, nos quais predominaram a pesquisa bibliográfica e a análise de dados secundários (Tabela 4).

Tabela 4 - Perfil metodológico

\begin{tabular}{|l|r|r|r|r|r|r|r|r|r|}
\hline \multirow{2}{*}{ Perfil metodológico } & \multirow{2}{*}{ Geral } & \multicolumn{7}{|c|}{ Eixos temáticos } \\
\cline { 3 - 10 } & & $\mathbf{1}$ & \multicolumn{1}{c|}{$\mathbf{2}$} & \multicolumn{1}{c|}{$\mathbf{3}$} & $\mathbf{4}$ & \multicolumn{1}{c|}{$\mathbf{5}$} & \multicolumn{1}{c|}{} & \multicolumn{1}{c|}{7} & $\mathbf{8}$ \\
\hline Teórico & $29 \%$ & $35 \%$ & $40 \%$ & $25 \%$ & $33 \%$ & $22 \%$ & $14 \%$ & 0 & $11 \%$ \\
\hline Teórico-empírico & $71 \%$ & $65 \%$ & $60 \%$ & $75 \%$ & $67 \%$ & $78 \%$ & $86 \%$ & $100 \%$ & $89 \%$ \\
\hline Empírico & 0 & 0 & 0 & 0 & 0 & 0 & 0 & 0 & 0 \\
\hline Total & $\mathbf{1 0 0} \%$ & $\mathbf{1 0 0} \%$ & $\mathbf{1 0 0} \%$ & $\mathbf{1 0 0} \%$ & $\mathbf{1 0 0} \%$ & $\mathbf{1 0 0} \%$ & $\mathbf{1 0 0} \%$ & $\mathbf{1 0 0} \%$ & $\mathbf{1 0 0} \%$ \\
\hline
\end{tabular}

Os resultados específicos referentes a cada eixo temático acompanham os gerais, com muitos artigos teórico-empíricos e somente alguns teóricos. A exceção é o tema gestão de gerações (7), que apresenta 100\% dos artigos com perfil metodológico teórico-empírico. Também merece destaque o tema gestão da diversidade (2), que possui uma maior concentração de estudos puramente teóricos. 


\subsection{Abordagem metodológica}

Apenas para os artigos que apresentaram perfil metodológico teórico-empírico (86 artigos), foram analisadas a abordagem e a estratégia metodológica utilizadas para a realização do estudo empírico. Em relação à abordagem metodológica, a tabela 5 mostra que a maioria desses artigos foi quantitativa (51\%). A abordagem qualitativa foi utilizada em 30\% dos artigos, e o uso combinado das duas abordagens aconteceu em 19\%.

Tabela 5 - Abordagem metodológica

\begin{tabular}{|l|r|r|r|r|r|r|r|r|r|}
\hline \multirow{2}{*}{ Abordagem metodológica } & \multirow{2}{*}{ Geral } & \multicolumn{7}{|c|}{ Eixos temáticos } \\
\cline { 3 - 10 } & & $\mathbf{1}$ & \multicolumn{1}{c|}{$\mathbf{2}$} & $\mathbf{3}$ & $\mathbf{4}$ & \multicolumn{1}{c|}{$\mathbf{5}$} & \multicolumn{1}{c|}{$\mathbf{c}$} & \multicolumn{1}{c|}{$\mathbf{7}$} & $\mathbf{8}$ \\
\hline Qualitativa & $30 \%$ & $18 \%$ & $22 \%$ & $33 \%$ & $35 \%$ & $43 \%$ & $33 \%$ & $100 \%$ & $25 \%$ \\
\hline Quali-quantitativa & $19 \%$ & $6 \%$ & $33 \%$ & 0 & $23 \%$ & $14 \%$ & $50 \%$ & 0 & $13 \%$ \\
\hline Quantitativa & $51 \%$ & $76 \%$ & $44 \%$ & $67 \%$ & $42 \%$ & $43 \%$ & $17 \%$ & 0 & $63 \%$ \\
\hline Total & $\mathbf{1 0 0} \%$ & $\mathbf{1 0 0} \%$ & $\mathbf{1 0 0} \%$ & $\mathbf{1 0 0} \%$ & $\mathbf{1 0 0} \%$ & $\mathbf{1 0 0} \%$ & $\mathbf{1 0 0} \%$ & $\mathbf{1 0 0} \%$ & $\mathbf{1 0 0} \%$ \\
\hline
\end{tabular}

A análise das frequências específicas de cada eixo temático permite visualizar exceções, como o tema gestão de gerações (7), que apresenta 100\% de artigos com abordagem qualitativa, e o tema responsabilidade corporativa (6), no qual a metade dos artigos é de abordagem quali-quantitativa.

\subsection{Estratégia metodológica}

Em relação à estratégia metodológica, os artigos de perfil teórico-empírico utilizaram, com maior frequência, a estratégia survey (48\%), seguida da estratégia de estudo de caso (22\%), sendo que, em $5 \%$ dos casos, as duas estratégias foram utilizadas de forma combinada. Além disso, também foram adotadas as entrevistas (8\%) e a observação (3\%). Outras estratégias foram empregadas em 14\% dos artigos (grupos focais e análise de dados secundários (Tabela 6)).

Tabela 6 - Estratégia metodológica

\begin{tabular}{|l|r|r|r|r|r|r|r|r|r|}
\hline \multirow{2}{*}{ Estratégia metodológica } & \multirow{2}{*}{ Geral } & \multicolumn{7}{|c|}{ Eixos temáticos } \\
\cline { 3 - 10 } & & $\mathbf{1}$ & \multicolumn{1}{c|}{$\mathbf{2}$} & \multicolumn{1}{c|}{$\mathbf{3}$} & $\mathbf{4}$ & $\mathbf{5}$ & \multicolumn{1}{c|}{$\mathbf{7}$} & \multicolumn{1}{c|}{$\mathbf{7}$} & \multicolumn{1}{c|}{$\mathbf{8}$} \\
\hline Survey & $48 \%$ & $53 \%$ & $67 \%$ & $67 \%$ & $58 \%$ & $14 \%$ & $17 \%$ & $33 \%$ & $63 \%$ \\
\hline Estudo de caso & $22 \%$ & $18 \%$ & $22 \%$ & $33 \%$ & $23 \%$ & $29 \%$ & $17 \%$ & $33 \%$ & $13 \%$ \\
\hline Observação & $3 \%$ & 0 & 0 & 0 & 0 & $21 \%$ & 0 & 0 & 0 \\
\hline Pesquisa bibliográfica & 0 & 0 & 0 & 0 & 0 & 0 & 0 & 0 & 0 \\
\hline Survey com estudo de caso & $5 \%$ & 0 & 0 & 0 & $8 \%$ & 0 & $33 \%$ & 0 & 0 \\
\hline Entrevistas & $8 \%$ & $12 \%$ & 0 & 0 & $8 \%$ & $7 \%$ & $17 \%$ & $33 \%$ & $13 \%$ \\
\hline Outros & $14 \%$ & $18 \%$ & $11 \%$ & 0 & $4 \%$ & $29 \%$ & $17 \%$ & 0 & $13 \%$ \\
\hline Total & $\mathbf{1 0 0} \%$ & $\mathbf{1 0 0} \%$ & $\mathbf{1 0 0} \%$ & $\mathbf{1 0 0} \%$ & $\mathbf{1 0 0} \%$ & $\mathbf{1 0 0} \%$ & $\mathbf{1 0 0} \%$ & $\mathbf{1 0 0} \%$ & $\mathbf{1 0 0} \%$ \\
\hline
\end{tabular}

As frequências analisadas por cada eixo temático são similares às do resultado geral dos artigos. Uma das exceções é o tema aprendizagem organizacional (5), que tem a estratégia de estudo de caso com maior frequência (29\%) que a survey (14\%) e que usou outras estratégias 
em 29\% dos casos. Já o tema responsabilidade corporativa (6) tem maior frequência de artigos que juntam as estratégias survey e o estudo de caso (33\%).

\subsection{Conteúdo dos trabalhos}

O conteúdo dos trabalhos foi bastante diversificado, em virtude da própria variedade de objetivos apresentados nos diferentes artigos investigados. Por outro lado, as análises realizadas permitem apresentar brevemente os resultados mais frequentes e mais importantes dentro de cada temática. No campo da gestão estratégica de pessoas, vários estudos comprovaram as associações entre gestão de pessoas e resultados organizacionais, alguns pautados sobre a perspectiva universalista, que defende a existência de melhores práticas, capazes de gerar melhor desempenho em qualquer contexto; outros pautados sobre as abordagens contingencial e configuracional, ressaltando a importância dos alinhamentos vertical e horizontal, respectivamente, para maximizar os benefícios proporcionados pelo sistema de gestão de pessoas. Alguns deles detalham características específicas, que podem contribuir, mais fortemente, com o nível de desempenho organizacional, tais como: configuração de gestão de pessoas baseada no comprometimento (CHOW; HUANG; LIU, 2008), trabalho em equipe (PROCTER; BURRIDGE, 2008), culturas organizacionais com ênfase na flexibilidade (WEI et al., 2008), estratégia de diferenciação e estrutura descentralizada (CAMPS; LUNA-AROCAS, 2009).

Os artigos que tratam da gestão da diversidade apresentam resultados bastante variados. Entre eles, é possível citar as evidências de que pequenas empresas possibilitam maior inclusão de gays e lésbicas (DAY; GREENE, 2008) e que a implantação de práticas efetivas de gestão da diversidade torna as organizações mais atraentes para as mulheres e minorias, além de atrair profissionais altamente qualificados (NG; BURKE, 2005). Também é importante salientar a relevância de desenvolver uma cultura organizacional inclusiva, com ênfase na transformação da cultura e da atitude, o que requer que os gestores e executivos ultrapassem barreiras, deixando de gerir a diversidade para passar a gerir em prol da diversidade (CHAVEZ; WEISINGER, 2008).

Em virtude do pequeno número de artigos encontrados na área de gestão de talentos, não é possível comentar os resultados gerais mais frequentes. Entretanto, vale destacar o estudo de Hausknecht, Rodda e Howard (2009), que identifica os fatores mais importantes para a retenção de talentos nas organizações, a saber: satisfação com o trabalho, remuneração extrínseca, relações no trabalho, compromisso organizacional, entre outros.

As publicações de gestão de pessoas internacional têm como pontos principais de conclusão a importância estratégica da gestão de pessoas no processo de internacionalização de empresas, a dificuldade do processo de expatriação, entre outros. Em alguns artigos, são apontadas as dificuldades encontradas para a atração, motivação e retenção dessas pessoas em função da mobilidade internacional e da globalização (MEYSKENS et al., 2009). Além disso, o principal fator demonstrado como determinante para essa mobilidade é o trade-off entre a empresa e a família nesses cargos internacionais. Quanto ao processo de expatriação, um dos fatores mais apontados para o êxito deste processo é o suporte de carreira (HEIJDEN; ENGEN; PAAUWE, 2009), sendo que a volta para casa é a parte mais importante do processo de expatriação (STARR, 2009).

Com relação às políticas e práticas adotadas nas subsidiárias, a conclusão apresentada em alguns artigos é de que, quanto mais os países da central e da subsidiária estão distantes culturalmente, maior a preferência por manter um grande número de funcionários expatriados da matriz, devido ao impacto negativo dessas diferenças na performance dessas operações internacionais (COLAKOGLU; CALIGIURI, 2008). Outros artigos demonstram a importância de configurações específicas das práticas em gestão internacional de RH para a retenção de trabaIhadores locais em plantas de multinacionais (REICHE, 2007). 
A principal questão abordada e concluída nos artigos de aprendizagem organizacional está associada à importância do contexto para incentivar o aprendizado, a criação e a transferência de conhecimento. Nesse sentido, o RH é apontado como agente ativo na captação de talentos (FELIN; ZENGER; TOMSIK, 2009) e na manutenção de capital social (YAMAO; DE CIERI; HUTCHINGS, 2009). De maneira geral, a interpretação predominante é a de que pressões e oportunidades do ambiente podem estimular os diferentes tipos de aprendizado e dar suporte a eles, e que o RH pode e deve favorecer esse processo (LONDON; SESSA, 2007).

Os estudos sobre responsabilidade corporativa constataram que a gestão de pessoas assume um importante papel nas organizações que pretendem operar de forma sustentável (JABBOUR; SANTOS, 2008), e que a responsabilidade social empresarial possui impactos positivos sobre a percepção dos funcionários (FURTADO; PENA, 2006), o comprometimento organizacional (BRAMMER; MILLINGTON; RAYTON, 2007) e sobre a sua capacidade de atrair talentos (FARIA; FERREIRA; CARVALHO, 2008). Verificou-se ainda que a motivação para a responsabilidade social parece estar mais associada aos resultados econômicos, com um caráter instrumental e com ações impulsionadas pelas forças de mercado e pelo atendimento às expectativas dos stakeholders (MATTONI; PENA; QUEIROZ, 2007).

Os resultados apresentados nos artigos sobre gestão de gerações abordam, de forma muito específica, os aspectos associados ao envelhecimento da força de trabalho e à aposentadoria. Gardiner et al. (2007), por exemplo, destacam que transições de emprego e aposentadoria em indivíduos por volta dos 50 a 60 anos estão ocorrendo em um contexto de grandes mudanças, que terão grande impacto sobre o mercado e o ambiente de trabalho nas próximas três décadas.

Entre os principais resultados associados aos artigos sobre modalidades de trabalho flexíveis, vale destacar que o contexto de competição tem pressionado as organizações a adotar formas de trabalho flexíveis. Esta pressão cria uma relação complexa para as organizações, com relações positivas entre o trabalho flexível e as percepções dos funcionários sobre a qualidade do trabalho em curto prazo, ao passo que, em longo prazo, existem custos envolvidos no que se refere às expectativas de desenvolvimento e progresso na carreira (KELLIGER; ANDERSON, 2008). O trabalho de Michie e Sheehan (2005) é conclusivo de que organizações com estratégia de redução de custos conseguem um retorno maior ao recorrerem a contratos de trabalho flexíveis em situações de necessidade sazonal de mão de obra, enquanto organizações com estratégia de inovação e melhoria da qualidade necessitam de contratos formais de trabalho, para preservar o colaborador por mais tempo na empresa, permitindo a formação da memória organizacional e o alinhamento estratégico.

\section{CONSIDERAÇÕES FINAIS}

O artigo apresentou o resultado da investigação acadêmica nacional e internacional sobre oito temas emergentes em gestão de pessoas levantados a partir de uma prévia revisão da literatura. Os temas foram destacados por serem recorrentes nos estudos e pesquisas sobre tendências na área. A análise dos artigos investigados apontou os temas gestão de pessoas internacional, gestão estratégica de pessoas, aprendizagem organizacional e gestão da diversidade, respectivamente, como os mais frequentes dentro de método utilizado.

Com relação aos aspectos metodológicos, a abrangência analítica mais frequente foi a nacional (32\%), mas alguns estudos também abordaram mais de um país (12\%). Já a natureza da maioria das pesquisas investigadas foi a exploratória (59\%), e o perfil metodológico predomi- 
nante, nos estudos, foi o teórico-empírico. Além disso, a abordagem predominante foi a quantitativa, com o uso da estratégia metodológica survey (48\%) e do estudo de caso (22\%) na maior parte dos trabalhos. Considerando a diversidade de temas e estratégias metodológicas, os objetivos também foram diversificados. Assim, a combinação de diferentes objetivos, em diferentes temas e métodos, resultou em uma grande diversidade de resultados. No entanto, por meio de análises, alguns resultados podem ser destacados.

No campo da gestão estratégica de pessoas, vários estudos comprovaram as associações entre gestão de pessoas e resultados organizacionais. Na gestão da diversidade, foi importante a constatação de que a implantação de práticas efetivas de gestão da diversidade torna as organizações mais atraentes para as mulheres e minorias, além de atrair profissionais altamente qualificados. As publicações de gestão de pessoas internacional têm como um dos pontos principais de conclusão a importância estratégica da gestão de pessoas no processo de internacionalização de empresas. Nos artigos de aprendizagem organizacional, a principal questão abordada e concluída está ligada à importância do contexto para incentivar o aprendizado, a criação e a transferência de conhecimento. Os estudos sobre responsabilidade corporativa constataram que a gestão de pessoas assume um importante papel nas organizações que pretendem operar de forma sustentável. Entre os principais resultados associados aos artigos sobre modalidades de trabalho flexíveis, está o fato de que o contexto de competição tem pressionado as organizações a adotar formas de trabalho flexíveis. Os estudos sobre gestão de talentos e gestão de gerações não puderam ter seus resultados destacados, pois foram encontrados poucos artigos nestes temas e todos muito diversos.

A análise dos artigos mostra que as diferentes áreas temáticas realmente parecem ser de grande relevância para os desafios e as oportunidades que as organizações enfrentam atualmente. Na maior parte deles, a gestão de pessoas é fator crítico de sucesso para a busca de soluções, bem como para a implantação de novas estratégias e a busca de novos mercados.

\section{REFERÊNCIAS BIBLIOGRÁFICAS}

BRAMMER, S.; MILLINGTON, A.; RAYTON, B. The contribution of corporate social responsibility to organizational commitment. The International Journal of Human Resource Management, v. 18, n. 10, p.1.701-1.719, 2007.

BRANDÃO, H. P. Aprendizagem e competência nas organizações: uma revisão crítica de pesquisas empíricas. Revista Eletrônica de Gestão Organizacional, v. 6, n. 3, p. 321-342, 2008.

CAMPS, J.; LUNA-AROCAS, R. High involvement work practices and firm performance. The International Journal of Human Resource Management, v. 20, n. 5, p. 1.056-1.077, 2009.

CARDOSO, R. L. et al. Pesquisa científica em contabilidade entre 1990 e 2003. RAE, v. 45, n. 2, p. 34-45, abr./jun. 2005.
CHAVEZ, C. I.; WEISINGER, J. Y. Beyond diversity training: a social infusion for cultural inclusion. Human Resource Management, v. 47, n. 2, p. 331350, 2008.

CHOW, I. H.; HUANG, J. C.; LIU, S. Strategic HRM in China: configurations and competitive advantage. Human Resource Management, v. 47, n. 4, p. 687 706, 2008.

COLAKOGLU, S.; CALIGIURI, P. Cultural distance, expatriate staffing and subsidiary performance: the case of US subsidiaries of multinational corporations. The International Journal of Human Resource Management, v.19, n. 2, p. 223-239, 2008.

DAY, N. E.; GREENE, P. G. A case for sexual orientation diversity management small and large organizations. Human Resource Management, v. 47, n. 3, p. 637-654, 2008. 
DEADRICK, D. L.; GIBSON, P. A. Revisiting the research-practice gap in HR: a longitudinal analysis. Human Resource Management Review, v. 19, n. 2, p. 144-153, 2009.

EGRI, C. P.; RALSTON, D. A. Corporate responsibility: a review of international management research from 1998 to 2007. Journal of International Management, v. 14, p. 319-339, 2008.

EUROFOUND (European Foundation for the Improvement of Living and Working Conditions). Europe at work: better life and opportunities for all. Dublin/Ireland. Four-year work programm: 20092012.

FARIA, M. D. de; FERREIRA, D. A.; CARVALHO, J. L. F. Responsabilidade Social Empresarial: um fator de atração para novos talentos? In: EnANPAD, 32. 2008, Rio de Janeiro. Anais... Rio de Janeiro: ANPAD, 2008. p. 1-16.

FELIN, T.; ZENGER, T. R.; TOMSIK, J. The knowledge economy: emerging organizational forms, missing microfoundations, and key considerations for managing Human Capital. Human Resource Management, v. 48, n. 4, 2009. p. 555-570.

FRIEDMAN, B. A. Globalization implications for human resource management roles. Employ Respons Rights J., v. 19, p. 157-171, 2007.

FURTADO, R. A.; PENA, R. P. M. Responsabilidade Social Empresarial com o Público Interno: a Percepção dos Empregados da Promon. In: EnANPAD, 30., 2006, Salvador. Anais... Salvador: ANPAD, 2006. p. $1-17$.

GARDINER, J. et al. Work-life balance and older workers: employees' perspectives on retirement transitions following redundancy. The International Journal of Human Resource Management, v. 18, n. 3, p. 476-489, 2007.

HAUSKNECHT, J. P.; RODDA, J; HOWARD, $M$. Targeted employee retention: performance-based and job-related differences in reported reasons for staying. Human Resource Manegement, v. 48, n. 2, p. 269-288, 2009.

HEIJDEN, J. A. V.; ENGEN, M. L.; PAAUWE, J. Expatriate career support: predicting expatriate turnover and performance. The International Journal of Human Resource Management, v. 20, n. 4, p. 831-845, 2009.
HOPPEN, N.; MEIRELLES, F. S. Sistemas de informação: um panorama da pesquisa científica entre 1990 e 2003. RAE, v. 45, n. 1, p. 24-35, jan./mar. 2005.

ISI. ISI Web of Knowledge. Disponível em: < http:/ /images.isiknowledge.com/WOK46/help/WOK/ h_database.html\#ccr>. Acesso em: 29 jun. 2009.

JABBOUR, C. J. C.; SANTOS, F. C. A.; BARBIERI, J. C. Gestão ambiental empresarial: um levantamento da produção científica brasileira divulgada em periódicos da área de administração entre 1996 e 2005. RAC, Curitiba, v. 12, n. 3, p. 689-715, jul./set. 2008.

JABBOUR, C. J. C.; SANTOS, F. C. A. The central role of human resource management in the search for sustainable organizations. The International Journal of Human Resource Management, v. 19, n. 12, p. 2.133-2.154, 2008.

KELLIGER, C.; ANDERSON, D. For better or worse? An analysis of how flexible working practices influence employee's perceptions of job quality. The International Journal of Human Resource Management, v. 19, n. 3, 2008. p. 419-431

KERSLEY, B. et al. Inside the workplace: first findings from the 2004 workplace employment relations survey. Great Britain: Department of Trade and Industry, 2005.

LAUTSCH, B. A.; SCULLY, M. A. Restructuring time: Implications of work-hours reductions for the working class. Human Relations, v. 60, n. 5, p. 719-743, 2007.

LENGNICK-HALL, M. L. et al. Strategic human resource management: the evolution of the field. Human Resource Management Review, v. 19, n. 2, p. 64-85, 2009.

LEPAK, D. P.; SHAW. J. D. Strategic HRM in North America: looking to the future. The International Journal of Human Resource Management, v. 19, n. 8, p. 1.486-1.499, ago. 2008.

LONDON, M.; SESSA, V. I. How groups learn, continuously. Human Resource Management, v. 46, n. 4, p. 651-669, 2007. 
LOSEY, M.; MEISINGER, S. R.; ULRICH, D. Conclusion: reality, impact and professionalism. Human Resource Management, v. 44, n. 2, p. 201206, summer. 2005.

MARTÍN-ALCÁZAR, F.; ROMERO-FERNÁDEZ, P. M.; SÁNCHEZ-GARDEY, G. Human resource management as a field of research. British Journal of Management, v. 19, n. 2, p. 103-119, jun. 2008.

MATTONI, L. A. M.; PENA, R. P. M.; QUEIROZ, H. M. Responsabilidade social empresarial e estratégia: estudo de caso sobre a gestão do público interno em empresa signatária do Global Compact. In: EnANPAD, 31., 2007, Rio de Janeiro. Anais... Rio de Janeiro: ANPAD, 2007. p. 1-16.

MEYSKENS, M. et al. The paradox of international talent: alternative forms of international assignments. The International Journal of Human Resource Management, v. 20, n. 6, p. 1.439-1.450, 2009.

MICHIE, J.; SHEEHAN, M. Business strategy, human resources, labour market flexibility and competitive advantage. The International Journal of Human Resource Management, v. 16, n. 3, p. 445-464, mar. 2005.

MORLEY, M. J. Contemporary debates in European human resource management: Context and content. Human Resource Management Review, v. 14, p. 353-364, 2004.

NG, E. S. W.; BURKE, R. J. Person-organization fit and the war for talent: does diversity management make a difference? The International Journal of Human Resource Management, v. 16, n. 7, p. 1.195-1.210, 2005.

NISHII, L. H.; ÖZBILGIN. M. F. Global diversity management: towards a conceptual framework. The International Journal of Human Resource Management, v. 18, n. 11, p. 1.883-1.894, nov. 2007.

PROCTER, S.; BURRIDGE, M. Teamworking and performance: the extent and intensity of teamworking in the 1998 UK Workplace Employee Relations - Survey (WERS98). The International Journal of Human Resource Management, v. 19, n. 1, p. 153-168, 2008.
REICHE, B. The effect of international staffing practices on subsidiary staff retention in multinational corporations. The International Journal of Human Resource Management, v. 18, n. 4, p. 523-536, 2007.

ROSSONI, L. A dinâmica de relações no campo da pesquisa em organizações e estratégia no Brasil: uma análise institucional. 2006.296 f. Dissertação (Mestrado em Administração) - Centro de Pesquisa e Pós-Graduação em Administração da Universidade Federal do Paraná, Curitiba, 2006.

SCHOLL, W. et al. The Future of Knowledge management: an international Delphi study. Journal of Knowledge Management, v. 8, n. 2, p. 19-35, 2004.

SHEN, J. et al. Managing diversity through human resource management: an international perspective and conceptual framework. The International Journal of Human Resource Management, v. 20, n. 2, p. 235-251, fev. 2009.

SHRM. Society for Human Resource Management. The 2007-2008 workplace trends list: the top trends according to SHRM's Special Expertise Panels, 2007.

. Workplace forecast, jun. 2008.

STARR, T. L. Repatriation and short-term assignments: an exploration into expectations, change and dilemmas. The International Journal of Human Resource Management, v. 20, n. 2, p. 286-300, 2009.

TONELLI, M. J. et al. Produção acadêmica em recursos humanos no Brasil: 1991-2000. RAE, v. 43, n. 1, p. 105-122, jan./mar. 2003.

WEI, L. Q. et al. The role of corporate culture in the process of strategic human resource management: evidence from chinese enterprises. Human Resource Management, v. 47, n. 4, p. 777-794, 2008.

YAMAO, S.; CIERI, H.; HUTCHINGS, K. Transferring subsidiary knowledge to global headquarters: subsidiary senior executives' perceptions of the role of HR configurations in the development of knowledgestocks. Human Resource Management, v. 48, n. 4, p. 531-554, 2009. 